Supporting Information for

A Trace Amount of Surfactants Enables Diffusiophoretic Swimming of Bacteria

Viet Sang Doan, Prakit Saingam, Tao Yan, and Sangwoo Shin

\title{
List of movies
}

Movie S1: Diffusiophoretic migration of $E$. coli into dead-end pores by $\mathrm{NaCl}$ gradient. In the absence of $\mathrm{NaCl}$ gradient (inner solution concentration $c_{i}=$ outer concentration $\left.c_{o}=1 \mathrm{mM}\right), E$. coli (EC) does not enter the pore, whereas in the presence of $\mathrm{NaCl}$ gradient $\left(c_{i}=100 \mathrm{mM}, c_{o}=1\right.$ $\mathrm{mM}$ ), they migrate actively into the pores. The play speed is $30 \times$.

Movie S2: Diffusiophoretic migration of various bacteria into dead-end pores by $\mathrm{NaCl}$ gradient $\left(c_{i}=100 \mathrm{mM}, c_{o}=1 \mathrm{mM}\right)$. Bacterial species include E. coli (EC), E. faecalis (EF), S. enterica (SE), and $V$. parahaemolyticus (VP). E. coli contains green fluorescent proteins (GFP) whereas other bacteria are tagged with membrane-specific dye (FM 1-43). The play speed is $30 \times$.

Movie S3: E. coli migrating into the dead-end pore by $\mathrm{NaCl}$ gradient with varying sodium dodecyl sulfate (SDS) concentration. The play speed is $30 \times$.

Movie S4: E. faecalis migrating into the dead-end pore by $\mathrm{NaCl}$ gradient with varying sodium dodecyl sulfate (SDS) concentration. The play speed is $30 \times$.

Movie S5: S. enterica migrating into the dead-end pore by $\mathrm{NaCl}$ gradient with varying sodium dodecyl sulfate (SDS) concentration. The play speed is $30 \times$.

Movie S6: $V$. parahaemolyticus migrating into the dead-end pore by $\mathrm{NaCl}$ gradient with varying sodium dodecyl sulfate (SDS) concentration. The play speed is $30 \times$.

Movie S7: Migration of E. coli by SDS gradients. Initially, the cells are suspended in a $0.3 \mathrm{M}$ sucrose solution inside the dead-end pores. Subsequently, $0.1 \mathrm{mM}$ of SDS solution is introduced to create SDS gradients. The play speed is $30 \times$.

Movie S8: Migration of E. coli by cetylpyridinium chloride (CPC) gradients. Initially, the cells are suspended in a $0.3 \mathrm{M}$ sucrose solution inside the dead-end pores. Subsequently, $0.1 \mathrm{mM}$ of CPC solution is introduced to create CPC gradients. The play speed is $30 \times$.

Movie S9: Same solute conditions as in Movie S8 except that E. coli are tagged with membranespecific dye (FM 1-43). The play speed is $30 \times$.

Movie S10: Migration of E. coli by cetrimonium bromide (CTAB) gradients. Initially, the cells are suspended in a $0.3 \mathrm{M}$ sucrose solution inside the dead-end pores. Subsequently, $0.1 \mathrm{mM}$ or 1 $\mathrm{mM}$ of CTAB solution is introduced to create CTAB gradients. The play speed is $30 \times$.

Movie S11: Rapid accumulation of E. coli near flow junctions by diffusiophoresis. In the presence of $\mathrm{NaCl}$ gradients, the top vertical channels contain $100 \mathrm{mM} \mathrm{NaCl}$ and the bottom horizontal channel contains $1 \mathrm{mM} \mathrm{NaCl}$. SDS $(0.1 \mathrm{mM})$ is present in the rightmost case. The play speed is $10 \times$. 
Movie S12: Diffusiophoretic migration of $V$. parahaemolyticus into dead-end pores by $\mathrm{NaCl}$ gradient $\left(c_{i}=100 \mathrm{mM}, c_{o}=1 \mathrm{mM}\right)$ with (left; fluorescence) or without (right; bright field) the membrane-specific dye (FM 1-43). The play speed is $30 \times$. 